\title{
HYDRAULIQUE
}

\section{La similitude dynamique et ses applications à l'hydraulique industrielle}

\author{
Par A. FOCH, Professeur à la Faculté des Sciences de Bordeaux.
}

Dans cel article, lauteur, après avoir exposć d'une facon gínérale, les principes de la similitule dynamique, indique les principales applications de la théorie à l'hydraulique industrielle: pertes de charges dans: les tuyaux et les coudes, formes des lignes de courant, pertes par vagues et remous. Il termine par des indications sur la similitude des mouvements dans une machine et son modèle.

I

Il y a longtemps que les hydrauliciens ont senti le besoin d'étudier, sur des modèles à échelle réduite, le rendement des installations qui devront être réalisées en grand. Pour ne prendre que l'exemple le plus connu, la théorie des turbines semblables, si magistralement développée par $\mathrm{M}$. Rateau, il y a trente ans, permet de choisir, entre tous les types possibles, le modèle le mieux adapté à des conditions locales déterminées, ou, pour parler plus exactement, de prévoir, d'après les essais faits sur une roue à échelle réduite, le rendement qu'offrirait la même rone établie pour une chute déterminée.

Or, d'innombrables questions se posent, dont la solution mathématique complète paraît devoir rester longtemps encore inconnue. L'étude expérimentale s'impose donc. Mais les facteurs qui peuvent influer sur la solution finale sont, en général, très nombreux; dans le cas cité plus haut des turbines à rendement maximum, il serait nécessaire d'étudier l'action de la hauteur de chute, du nombre de tours à la minute, du débit, de la forme, etc... Mais les théories de la similitude permettent souvent de réduire le nombre de ces facteurs : dans l'exemple précédent, on sait qu'il suffit de connaître, pour une roue déterminée, les valeurs du rendement en fonction du nombre de tours $n_{\mathrm{s}}$ à la minute, lorsque la turbine absorbe $1 \mathrm{~m}^{3} / \mathrm{sec}$. sous un mètre de chute, pour en déduire le rendement de toute turbine géomẹtriquement semblable absorbant $\mathrm{Q} \mathrm{\textrm {m } ^ { 3 }}$ : sec. sous $\mathrm{H} \mathrm{m}$. de chute et tournant à un nombre de tours $n$ tel que

$$
n_{\mathrm{s}}=\frac{n V \overline{\mathrm{Q}}}{\mathrm{I}^{\mathrm{i} / \mathrm{s}}}
$$

Grâce à la théorie, on a donc pu avoir une connaissance plus complète avec un nombre d'expériences bien plus restreint.

L'objet de cette étude est d'indiquer, d'une façon générale, et sur quelques exemples qui me paraissent assez typiques, les principes de la similitude mécanique (1). Ces principes sont, d'ailleurs, tous bien connus; mais, peut-être, n'est-il pas inutile de rassembler en quelques pages, ceux qui ont trait à la similitude hydraulique. La méthode que je suivrai est due, dans son principe, à Lord Rayleigh (2) ; d'autres existent (3), qui sont d'une apparence un peu plus générale. Personnellement, je les ai trouvées moins commodes, surtout parce que moins machinales.

II

Je rappelle ce que l'on est convenu d'appeler les dimensions d'une grandeur. Pour des raisons de commodité, il a été nécessaire d'établir entre les diverses grandeurs : longueur, masse,

(1) On consultera utilement le Dictionnory. of applied Physirs, de Glazebrook.

(2) Phil. Mag., 1899 (48), p. 321.

(3) Voir par exemple Helmtroltz, Wissenschafliche Abhandlungen tome I, p. 158.

Article published by SHF and available at http://www.sht-lhb.org or http://dx.dol.org/10.1051//hb/1923040 temps, force, quantité de chaleur, elc., des relations ; on a choisi certaines grandeurs comme fondamentales. De ces grandeurs fondamentales, les principes de la physique, les théorèmes rationnels et les lois expérimentales ont permis de déduire des unités dérivées. Par exemple, on prend comme unité d'aire le carrí construit sur l'unité de longueur ; comme unité de vitesse, li vitesse du mobile parcourant l'unité de longueur dans l'unité de temps. Le choix des unités fondamentales est d'ailleurs entie. rement arbitraire : ce peuvent ètre la longueur, la masse ct le temps, ou la longueur, la force et le temps, ou tout autre groupement. A l'heure actuelle, on s'accorde généralement à adopter le premier groupe : longueur, masse et temps.

Ce groupe une fois choisi, les unités dérivées sont déterminées ; par suite, le nombre qui mesure une vitesse changera avec les unités fondamentales ; par exemple, ce nombre, étant 60 si l'on prend comme unités fondamentales le kilomètre et l'heure, devient 500 si les unités fondamentales sont le pied et la seconde. Mais il est évident que les phénomènes eux-mèmes sont indipendants des appareils qui servent à les mesurer ; la chute d'un corps en un lieu déterminé reste la mème, que les déplacements soient mesurés avec le mètre des Archives ou le pied britannique. De façon précise, soit une grandeur quelconque (une force vive par exemple). Les définitions permettent de voir que si l'unite de longueur devient $a$ fois plus petile, l'unité de masse $b$ fois plus petile, l'unité de temps c fois plus petile, le nombre mesurant li grandeur deviendra

$$
a^{z} b^{\zeta} c^{\gamma}
$$

fois plus grand. Dans le cas de la force vive, on aurait :

$$
x=2, \quad \beta=1, \quad \gamma=-2
$$

On dit que les dimensions de la grandeur considérée sont:

$$
\text { L. M: }{ }^{\prime} \text { : }
$$

Comme toute expression mathématique d'une loi physique ne peut se traduire que par une égalité entre éléments de même nature, comme on ne peut ajouter entre elles une force et une énergie, une température et une vitesse, il faut nécessairement qute les divers termes de la relation soient des termes de même nalur, influencés par suite de la même façon par un changement d'unités. En d'autres termes, les divers termes doivent avoir mêmes dimensions. Par exemple, les divers termes de l'équation de Bernouilli

$$
z+\frac{\mathrm{P}}{\pi}+\frac{v^{2}}{2 g}=\mathrm{cle}
$$

ont même dimension $L$, puisque les dimensions de chacun des facteurs sont respectivement

$$
\begin{aligned}
& {[z]=1} \\
& |\mathrm{P}|=\mathrm{L}-1 \mathrm{MT}-2 \\
& |\pi|=\mathrm{L}-2 \mathrm{MT}-2 \\
& {[v]=\mathrm{LT}-1} \\
& {[g]=\mathrm{LT}-2}
\end{aligned}
$$


III

Or - el c'est là le point important - ce principe de l'homogénéilé, dans le sens étymologiçue du mot, peut servir non seujement pour contrôler l'exactitude d'un calcul, mais encore pour obtenir des renseignements à priori sur la forme à donner aux expressions expérimentales. Dans l'analyse préliminaire d'un problème déterminé, il lournit souvent un secours précieux pour indiquer la voie dans laquelle il convient de s'engager. L'exemple classique que je rappelle parce qu'il permet de préciser la méthode suivie et surtout la nature profonde de la similitude dynamique, est celui de la durée des petites oscillations d'un pendule simple. L'expérience montre que cette durée est indépendante de l'angle d'ecart ; par suite les seuls facteurs qui peuvent intervenir sont lit masse du pendule $m$, la longueur $l$ de ce pendule, l'intensité de la pesinteur g, la forme de la boule, le système de suspension. Les deux derniers facteurs paraissent difficiles à formuler par un paramètre (1). Commençons par les négliger, ou plus exactement supposons-les constants dans une série d'expériences.

Nous écrivons que la période f) est fonction de $m, l$ et $q$.

$$
0=l(m, l, g)
$$

.1 priori, la lonction $f$ est inconnue; supposons-la développée 'lll sćrie de puissances de $l, m$ et $g$.

$$
0=\Sigma \mathrm{A} l^{x} m l^{\beta} g{ }^{\prime}
$$

Chaçue terme doit avoir les dimensions d'un temps, en vertu du principe déjà rappelé, Donc, le terme général ayant comme dimensions

$$
\mathrm{L}^{\alpha} \mathrm{Mi}_{\mathrm{i}}\left(\mathrm{LT}^{-2}\right)
$$

on doit avoir :

$$
\begin{array}{r}
y=0 \\
x+y=0 \\
-2 y=1
\end{array}
$$

Donc :

$$
9=2 \mathrm{Am}^{0} l^{1} g^{-1}=-\frac{l}{g} \times B
$$

B étant une constante.

L'étude préliminaire nous permet done de voir qu'une seule crpérience sera nécessaire pour déterminer la valeur de $\mathrm{B}$, alors que l'étude purement expérimentale eût exigé la variation successive de $l, m$ et $g$. La simplification est évidente.

Allons plus loin ; cherchons le temps mis par le pendule pour aller de sa position d'équilibre à une position faisant un angle $x$ avec la verticale; nous avons à écrire comme plus haut que

$$
t=f(l, m, g, x)
$$

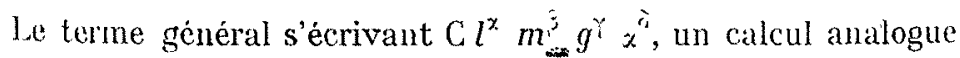
au précédent permet de voir que le terme doit s'écrire (un angle itant sans dimensions)

Done :

$$
\text { C } l^{\frac{1}{2}} g \stackrel{-1}{=} x^{8}
$$

$$
1=V_{g}^{l} \times \mathrm{LC} x^{\prime \prime}=V_{\frac{l}{d}}^{l}(x)
$$

Cette équation montre que l'angle pest détermini par la balentr du terme $1 \mathrm{~g}$

Photographions, sur des plaques de mème grandeur, deux

(1) Il s'agit d'une difficulté relative. On sait, en effet comment la considération des moments d'inertie résout complètement la question. pendules de longueurs $l_{1}$ et $l_{2}$, en des points où la gravité vaut. $g_{1}$ et $g_{2}$, à des époques $t_{1}$ et $t_{2}$ telles que :

$$
l_{1} \backslash \cdot \frac{g_{1}}{l_{1}}==l_{ \pm} \sqrt{\frac{g_{2}}{l_{2}}}
$$

Les deux séries de photographies ainsi obtenues seront identiques. Quand on peut réaliser des systèmes en mouvement passant par les mêmes états géométriques pour les mêmes valenrs d'un groupe mentsans dimensions (ici $\ell \sqrt{g}$, on dit que ces systèmes sont dynamiquement semblables (Hyman Lévy)".

\section{IV}

Cela étant, passons aux principaux problèmes de l'hydraulique industrielle que I'on peut aborder par la méthode des dimensions. Toutes les considérations qui vont suivre reposent sur la considération de l'équation générale.

$g \frac{1}{\partial} \frac{\partial p}{\partial x}+\frac{\partial u}{\partial t}+u \frac{\partial u}{\partial x}+v \frac{\partial u}{\partial y}+w \frac{\partial u}{\partial z}=\frac{\partial}{z}\left[\frac{\partial-u}{\partial x^{2}}+\frac{\partial{ }^{2} u}{\partial y^{2}}+\frac{\partial-1}{\partial z^{2}}\right]$ qui, jointe aux deux équations analogues et à l'équation d'incompressibilité

$$
\frac{\partial u}{\partial x}+\frac{\partial v}{\partial y}+\frac{\partial w}{\partial z}=0
$$

permettrait de calculer $p, u, v, w$, si on connaissant la densité $\vec{r}$ et la viscosité : du fluide, ainsi que les conditions aux limites. Il s'agit là d'une " permission " théorique ; les mathématiques sont, à l'heure actuelle, totalement incapables de donner à l'ingénieur le moyen de tracer à priori l'injecteur d'une roue Pelton ou le tube d'aspiration d'une turbine Francis. L'expérience doit forcément intervenir ; nous allons voir comment des considérations d'homogénéité permettent de restreindre le champ où les investigations semblent à prior $\grave{i}$ devoir s'étendre.

\section{1) Perte de charge dans les tuyaux.}

J'ai indiqué l'application de la méthode de Rayleigh à la perte de charge dans les tuyaux lisses dans la Houille Blanche $\left(\mathrm{n}^{\circ}\right.$ de septembre-octobre 1922). Pour être plus général, prenons le cas d'un tuyau rugueux et définissons la rugosité comme le rapport de la hauteur moyenne des rugosités $e$ au diamètre moyen du tuyau.

Cherchons la perte de charge en mètres d'eau par mètre de longueur du tuyau.

Nous écrirons cette perte (sans dimensions) :

$$
r_{i}=\frac{w^{2}}{2 \cdot g} f|w, i-,, c, d, e|
$$

$w$, vitesse moyenne de l'eau dans le tuyau. (dimensions LT-1) $u$, coefficient de viscosité absolue ........ ( $\left.-M^{-1} \mathrm{~T}^{-1}\right)$ $\hat{~}$, densité de l'eau ............... ( $\left.\mathrm{ML}^{-3}\right)$

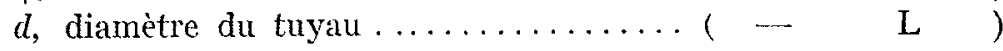

Nous exprimons ainsi la perte de charge en pour cent de la charge équivalente à la vitesse moyenne d'entrée.

Le terme général du développement en série de $r$ est :

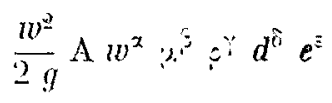

Il doit avoir comme dimensions 0 ; done :

$$
0=\mathrm{L} \times\left(\mathrm{LT}^{-1}\right)^{\alpha}\left(\mathrm{ML} \mathrm{L}^{-1} \mathrm{~T}-1\right)^{5}(\mathrm{ML}-3)^{r} \mathrm{~L}^{j} \mathrm{~L}^{\mathrm{s}}
$$

Donc :

$$
\begin{array}{cl}
\hat{y}+y=0 \\
2+x-3-3 y+\delta+z=1, & y=x \quad \hat{z}=-x+x-z \\
x+y=0 &
\end{array}
$$


Par suite le terme géuéral est de la forme:

$$
\frac{w^{2}}{2 y d}+\left(\frac{w d z}{y}\right)^{x}\left(\frac{c^{2}}{d}\right)^{3}
$$

ce qui montre que la perte de charge est de la forme :

$$
r_{i}=-\frac{w^{2}}{2} \frac{1}{g}+\frac{1}{d} f\left[\frac{w d}{2} \cdot \frac{e}{d}\right]
$$

$\checkmark$ etant la viscosite dynamique $\frac{0}{5}$

Ombeck a domné pour expression de $f\left[\frac{m d}{y}, \frac{e}{d}\right]$ une expression de la forme

$$
t=\mathrm{A}: \mathrm{B}_{\bar{d}}^{e} \cdot \overline{v d}
$$

A et B étant deux constantes; je n'insiste pas sur les valeurs numériques, en raison de la difficulté de définir avec précision la rugosité $\frac{e}{d}$; je me borne simplement à indiquer la voic où doit s'engager - et où s'est effectivement engagée - tout recherche rationnelle.

Jai signalé dans larticle déjà cité la splendide concordance avec la théorie précédente des résultats expérimentaux obtenus sur des fluides aussi différents que l'eau, l'air et la vapeur, Je me borne à indiquer que, pour les pertes de charge dans les tuyaux cvlindriques, la similitude drnamique d'un tuyau et de son modèle comporte la réalisation simultanée de légalité des quantités :

$$
\frac{b d}{y} \text { el } \frac{e}{d}
$$

Ces conditions réalisées, il en résultera que la perte de chargo relative $\frac{\unlhd h}{w^{2}}$ sera la même. Dans les tuvaux où la rugosité est $\overline{2 g}$

négligeable, il suffit de conserver la mème valeur pour $\frac{u d}{y}$

\section{Pertes de charge dans les coldes.}

Le raisonnement précédent est valable sans aucune modification. Soient done deux coudes semblables géométriquement; prenons la perte de charge $\lrcorner \mathrm{H}$ (en mètres d'eau) entre deux poinls du coude et entre les $d^{e_{u x}}$ points correspondants du modèle, nous trouvons que si $l$ est une dimension caractéristique du coude (par exemple le diamètre du premier tuyau), la perle de charge s'écrit :

$$
\left.\Delta \mathrm{H}=\frac{w^{2}}{2 g} / \mid \frac{w l}{\nu}, \frac{e}{d}\right\rceil
$$

$w$ étant la vitesse moyenne dans une section du coude. Si le coude peut être considéré comme lisse, on a :

$$
\Delta \dot{\mathrm{H}}=\frac{w^{2}}{2 g} F\left(\frac{w l}{y}\right)
$$

L'étude des pertes de charge dans les coudes comporterait done :

1) Une étude relalive aux coudes lisses, tels qu'on les peut, par exemple, réaliser en ciment;

2) Une étude de l'influence des rugosités (dues aux clouures, aux joints mal faits, etc.)

Cette étude doit être faite spécialement pour chaque formo de coude ; pour une forme déterminée, elle peut être faite sur un modèle unique, a condition de pouvoir réaliser une gamne sulfisamment étendue de vitesses (1).

\section{FORME DIS LIGNIS ME COLRANT.}

Pour en lerminer avec les phénomènes où intervient la viscosité supposons differents corps tous gémétriquement semblables. Dans un de ces corps caractérisé par me dimension particulière $l$ sécoule un litude: nous admeltrons réalise le mouvement permanent. La vilesse p en un point P' du fluide lixé par rapport au corps jecul, d'une façon générale, s'ecrire:

$$
b \cdots f_{1}(w, i, \nu, l)
$$

en appelant $"$ la vilesse mosemne déromlement, y el a la viscosité cinematicque el la densilé du lluide.

Soit, daulre part, ६. la pente de la ligne de courant passant par $\mathrm{P}^{\prime}$; nous ecrirons que.

$$
\overline{7}=f_{2}(l, \ldots, \%, l)
$$

v a pour dimensions L.T 1 , at de dimensions nulles, la méthode précédente montre que

$$
\begin{aligned}
& b \quad-\quad l i *\left(\frac{w l}{\%}\right) \\
& =-1 *\left(\begin{array}{c}
21) l \\
\frac{1}{2}
\end{array}\right)
\end{aligned}
$$

La premiere equalion montre que la vitesse p (en un point P) dépend uniquement de la position de ce point, de la vitesse moyenne et de $\frac{w l}{y}$; la pente dépend uniquement de la position du point el de $\frac{l w l}{\%}$. Si donc, on dispose de deux corps semblables, et si $\frac{w l}{y}$ est le mème pour ces deux corps :

1) Les lignes de courant seront semblables (au sens gèomtrique) :

2) Pour chacun de ces corps, si on peut faire varier la vitesse et la viscosité en maintenant constant $\frac{w l}{7}$, la vitesse de chacun des points sera proportionnelle a la vitesse moyemne.

De cela, une importante conséquence est à déduire. Certains des constructeurs de roues Pelton ont adopte pour tous leurs injecteurs, le même tracé ; d'autres constructeurs, au contraire, prétendent adapter leurs tracés à chaque cas particulier. Or, la théorie précédente établit que l'on ne peut, de façon stricte, conserver le mène tracé que si $\frac{b l}{\%}$ est constant. Supposons d'abord la lempérature de l'eau constante; deux injecteurs, géométriquement semblables, ont des débits proportionnels aux carrés des dimensions homologues et aux vitesses.

$$
\frac{q}{w l^{2}}=\frac{Q}{\mathrm{WL}}
$$

Mais on doit avoir, d'après ce qui précède :

Donc :

$$
w l=\mathrm{WI}
$$

$$
(7 l v)=Q \mathrm{~W}
$$

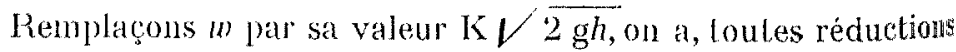
faites :

$$
\eta V h \cdots Q V \mathrm{H} \quad \text { oн } \frac{q h}{V \bar{h}}-\frac{\mathrm{QH}}{V \overline{\mathrm{II}}}
$$

(1) On sait que les comstructeurs athmettent pout i $\frac{w t}{y}$ une valeur de l'ordre de 1 quand il s'agil d'un coude brusque, une ratleur de lordre de $\frac{1}{100}$ pour les coudes progressifs 
İn d'autres termes, le tracé d'un injecteur ne serait valable sous une chute donnée que pour une puissance déterminée, laquelle puissance croîtrait comme la racine carrée de la chute.

Cela justifierait la pratique des derniers constructeurs.

Mais nous avons là une voie où il ne faut avancer que prudemment. On peul d'abord remarquer que le même raisonnement vaul pour les aujets et, à ma connaissance, aucun construcleur ne trace les modèles de ses aujets suivant de tels prin(ipes.

De plus, pour être rigoureux, on devrait laire intervenir la variation de viscosité de l'eau avec la température. Cetle varialion est relativement importante : de l'hiver à l'été, la viscosité diminue de plus de $50 \%$. A vouloir êlre strict, on doil donc conclure que le mème injecteur ne peut convenir également en temps de neige el au mois d'août. Toutefois, comme l'expérience montre que les bonnes roues Pelton ont des rendements assez constants quelle que soil l'époque de l'année, nous sommes amenes à penser que l'influence du $\frac{w l}{\%}$ sur la forme des lignes de courant n'est pas très grande. Est-elle négligeable? Cela est une autre question : Je citerai l'exemple d'une fourniture de l'ellon, pour très haule chules relativement puissantes, oì a clé obtemu un rendement de près de $5 \%$ supérieur aux rendements ordinaires. ce rendement, oblenu par loules les larbines de la fournilure, n'a plus élé retrouve depuis. Ne faudrait-il pas chercher la raison de ce fail dans la convenance particulièe des tracés du constructeur aux grandes puissances? Il ne sagit evidemment là que d'une suggestion. Néammoins, lintêrêt serait grand dune étude systématique du tracé des injecteurs d'une part et des angets de lautre. A l'heure ou lon "gratle "des $0,1 \%$ sur le rendement des appareils électriques, où l'exploitant paie $2 \%$ de plus pour que le rendement d'un transformateur passe de 0,975 a 0,98 , il ne serait peut-ètre pas inutile de savoir comment rattraper 5 à $6 \%$ sur les machines hydrauliques.

\section{$\gamma$}

1)ans les pages qui précèdent, nous avons considéré linfluence de la viscosité sur les mouvements des liquides. Nous allons maintenant considérer un cas très fréquent dans la pratique : celui de l'énergie perdue en vagues et remous dans les liquides dont on peut négliger la viscosité. Ce cas se rencontre dans les tubes d'aspiration au débouché dans le canal de fuite, dans certains rupteurs de charge faisant ćcouler l'eau sous pression d'un réservoir dans un canal à l'air libre, etc. Le phénomène peut s'analyser de ln façon suivante : leau arrive avec une certaine énergie cinćtique dans une masse d'eau considérable offrant un niveau à l'air libre. I'où naissance dans cette masse d'eau de vagues, de remous, de chocs ne s'amortissanl qu'à la longue. Pour établir les équations du plúnomène, on peut donc, en première approximation négliger la viscosité et se borner à l'énergie dépensée, à créer les mouvements de surface.

Les equations générales de 1'hydrodymamique prement la forme :

$$
\frac{\partial p}{\partial x}+\frac{\partial u}{\partial t}+u \frac{\partial u}{a x}+v \frac{\partial u}{\partial y}+m \frac{\partial u}{\partial z}=0
$$

avec deux épuations analogues. Ecrivant $p=h ; g$, il vient trois équations du type

$$
y \frac{a h}{a x}+\frac{\partial u}{\partial x}+u \frac{a u}{a x}+b \frac{a u}{\partial y}+u \frac{a u-0}{a z}-0
$$

et l'équation de contimuilé.

On voit que la hauteur d'eau au-desous d'un point domé dépend en particulier de l'accélération de la pesanteur ; on se rend comple aiscment de la raison de co dait : yuand une vague prend naissance quelque part, le niveau s'élève jusqu'à ce que l'énergie soit passée sous forme potentielle, la hauteur atteinte est d'autant plus grande que l'intenstié de la pesanteur est plus faible. Si, alors, nous considérons, par exemple, un tube d'aspiration et son modèle, nous aurons à écrire que l'énergie perdue dans l'un deux sera fonction, d'abord d'une longueur l caractérisant l'échelle (par exemple le diamètre du col à l'entrée), en second lieu de la vitesse en une région (par exemple la vitesse moyenne $w$ en ce col), et enfin de l'intensité de la pesanteur $g$. On peut toujours rapporter cette énergie à l'unité de débit et indiquer la hauteur de chute équivalente $h$. On pourra même chercher le rapport de celte dernière à l'énergie $\frac{w^{2}}{2 g}$ apportée dans le tube par l'unité de débit. En somme, sans restreindre en rien la généralité de l'expression, on peut écrire ;

$$
h=\frac{w^{2}}{2 g} f(w, l, g)
$$

Le terme général du déve'oppement de $f(u, l, g)$ est sans dimensions.

Ce terme élant écrit:

On doit avoir :

$$
A w^{z} l \ddot{ }
$$

$$
1=\left(\mathrm{LT}^{-1}\right)^{x} \mathrm{~L}^{\tilde{3}}(\mathrm{LT}-2)
$$

soit :

$$
\begin{aligned}
& 0=x+y+\gamma \\
& 0=x+2 y
\end{aligned}
$$

ou :

$$
\begin{aligned}
& r=-\frac{x}{2} \\
& y=-\frac{z}{2}
\end{aligned}
$$

Le terme général étant $A\left(\frac{w^{2}}{l g}\right)^{\frac{\alpha}{2}}$, on voit que la hauteur de chute perdue peut s'écrire :

$$
h=\frac{w^{2}}{2 g} f\left(\frac{w^{2}}{l g}\right)
$$

Ce qui peut s'interpréter ainsi : le rapport de l'énergie perdue à l'énergie transportée par le tube est fonction uniquement du rapport $\frac{w^{2}}{l q}$; en d'autres termes, les rendements d'un tube et de son modèle sonl les mêmes quand les vitesses sont dans le rapport des racines carrées des dimensions homologues (Règle de Froude).

Par conséquent, soit un tube daspiration ayant au col dentrée un dianètre de 2 mètres et débitant $25 \mathrm{~m}^{3}$ : sec; le modèle au $\frac{1}{20^{\mathrm{e}}}$ n'aura que 10 centimètres de diamètre au col d'entrée; dautre part, la vitesse derra y être réduite dans le rapport de 1 à $V \overline{20}$; lo débit dans le modele sera donc $\frac{1}{20^{2}} \times \frac{1}{120}$ fois plus petit que le débit réel : il suffira de $1,33 \mathrm{l}: \mathrm{sec}$. pour reproduire, $A \mathrm{Lx}$ rnotTEMENTs près, les phénomènes produits dans le tube réel.

Autre exemple. - Soit un lac fermé par un barrage-réservoir. Pour diverses raisons, il est impossible d'établir une conduite forcée à partir du barrage et l'on doit envoyer dans un canal d'amenée l'eau sortant du lac, à raison de $10 \mathrm{~m}^{3}$ : sec., sous une chute de 30 mètres. L'idéal serait évidemment d'utiliser cette puissance dans une turbine, mais nous admettons que la construction d'une usine au pied du barrage dépasse les possibilités financières du moment. Il faul donc detruire sur place une puissance de 4.000 C. V. Lides yui se présente toul naturellement est 


\begin{tabular}{|c|c|c|c|c|c|c|c|}
\hline $\mathrm{N}^{\mathrm{n}}$ & COURS D'EAU & NOMS DES USINES & $\begin{array}{l}\text { PROPRIETAIRES } \\
\text { POCATALS }\end{array}$ & PUISSANC & $\begin{array}{l}\text { NoRMiAI } \\
\text { S LSINFE } \\
\end{array}$ & $\mathrm{E} \mathrm{KW}$ & $\begin{array}{r}\text { PEEGIME } \\
\text { A- Autorisation. }\end{array}$ \\
\hline 1 & 2 & 3 & 4 & \begin{tabular}{|c} 
en \\
service \\
5 \\
\end{tabular} & $\begin{array}{l}\text { en cons- } \\
\text { truction } \\
6 \\
\end{array}$ & $\begin{array}{c}\text { en } \\
\text { projet } \\
1 \\
\end{array}$ & $\begin{array}{c}\text { sumementation. } \\
8\end{array}$ \\
\hline
\end{tabular}

\section{BASSIN DE L'ISÈRE (suite)}

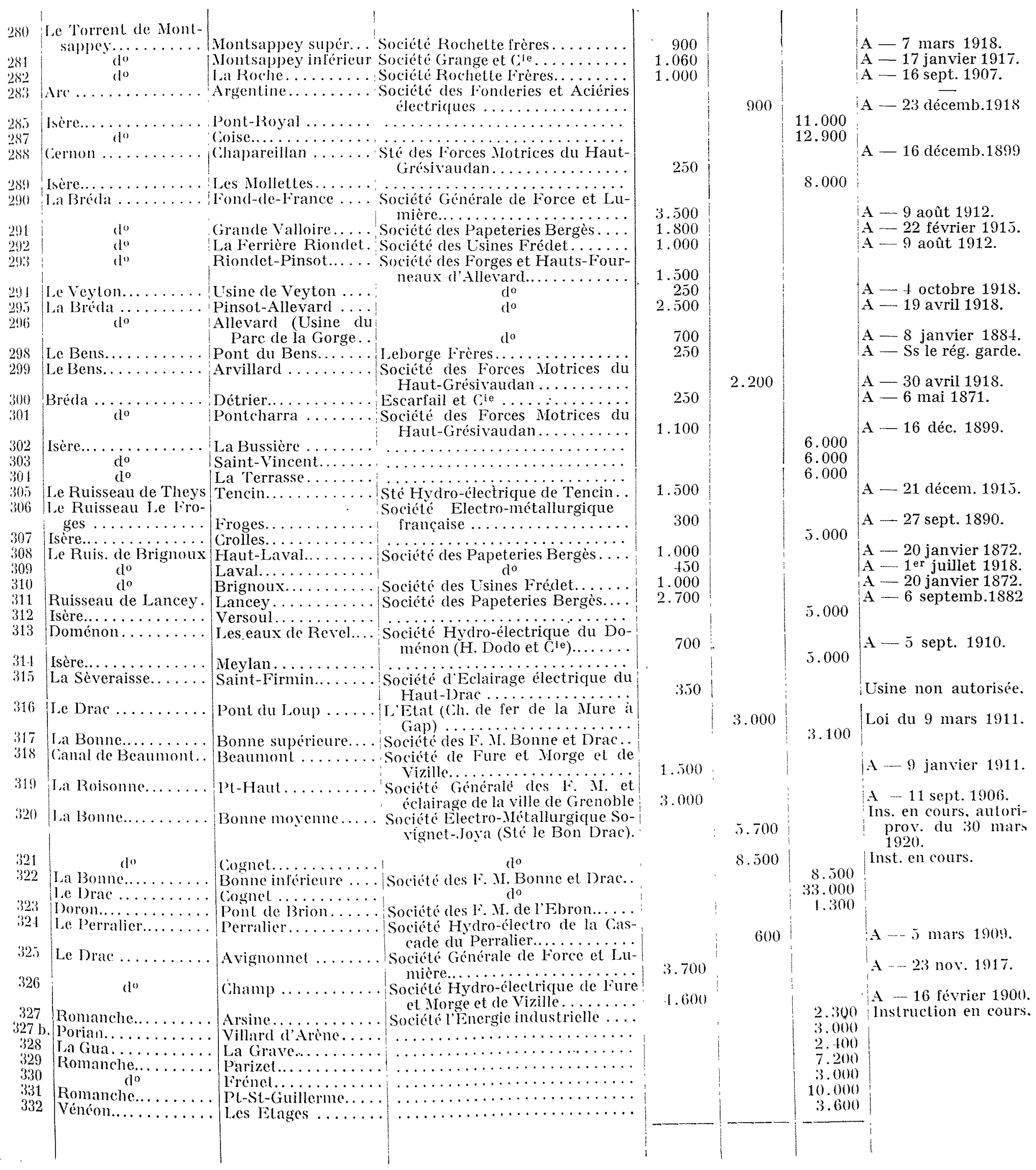




\begin{tabular}{|c|c|c|c|c|c|c|c|}
\hline No & 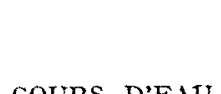 & NOUS DFS IISTYFS & PROPRIETAIRES & \multicolumn{3}{|c|}{$\begin{array}{c}\text { PUISSANCE NORMALE EN HW } \\
\text { DES USINES }\end{array}$} & $\begin{array}{r}\text { REGLME } \\
A-\text { Autorisation. }\end{array}$ \\
\hline d'ordre & LUUTS D LIA O & 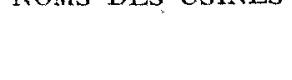 & LOCATAIRES ou EXPLOITANTS & $\begin{array}{c}\text { en } \\
\text { service }\end{array}$ & $\begin{array}{l}\text { en cons- } \\
\text { truction }\end{array}$ & $\begin{array}{c}\text { en } \\
\text { projet }\end{array}$ & $\begin{array}{l}\text { C - Concession et date do } \\
\text { réglementation. }\end{array}$ \\
\hline 1 & 2 & 3 & 4 & 5 & 6 & 7 & 8 \\
\hline
\end{tabular}

BASSIN DE L'ISĖRE (suite)

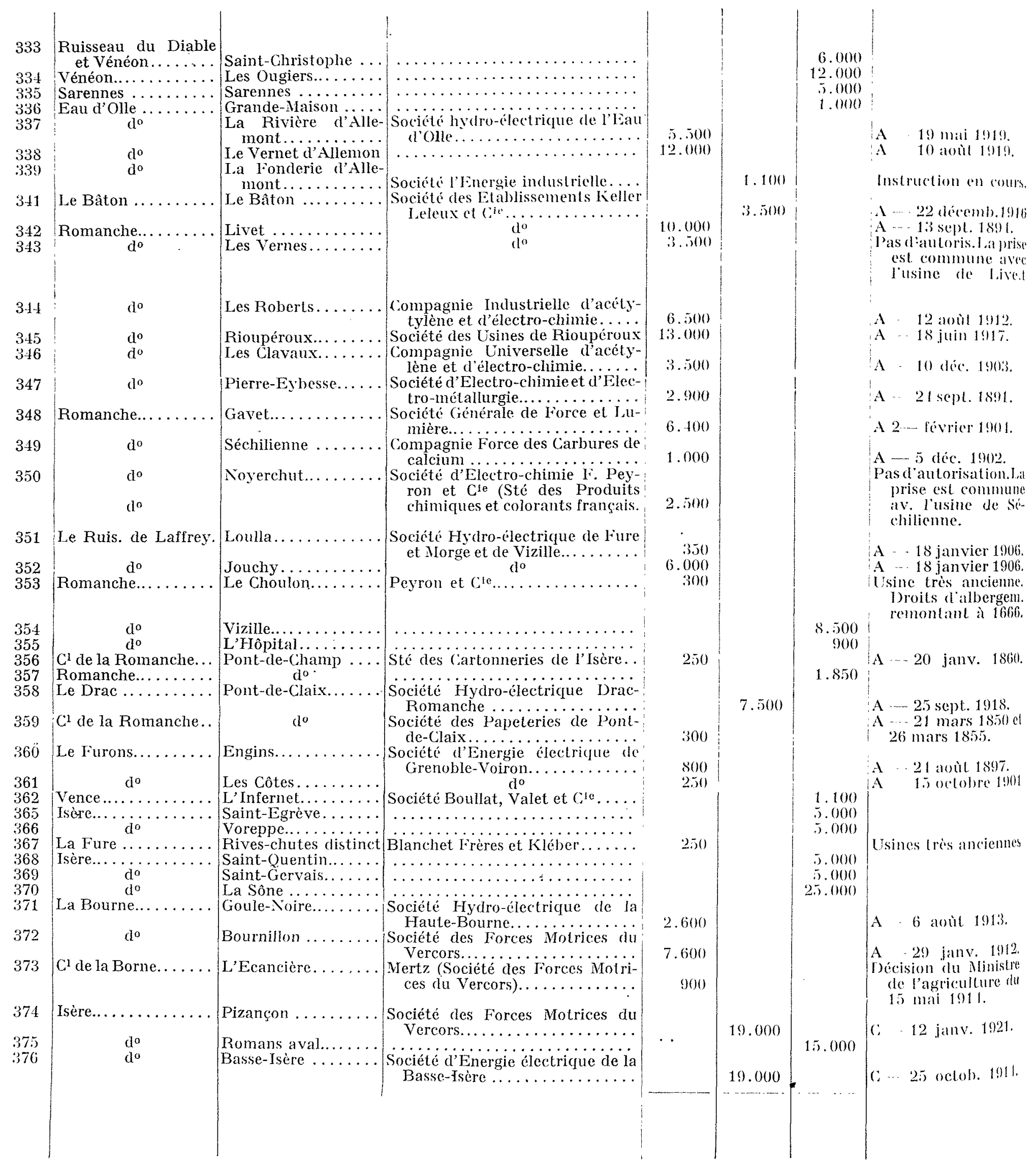




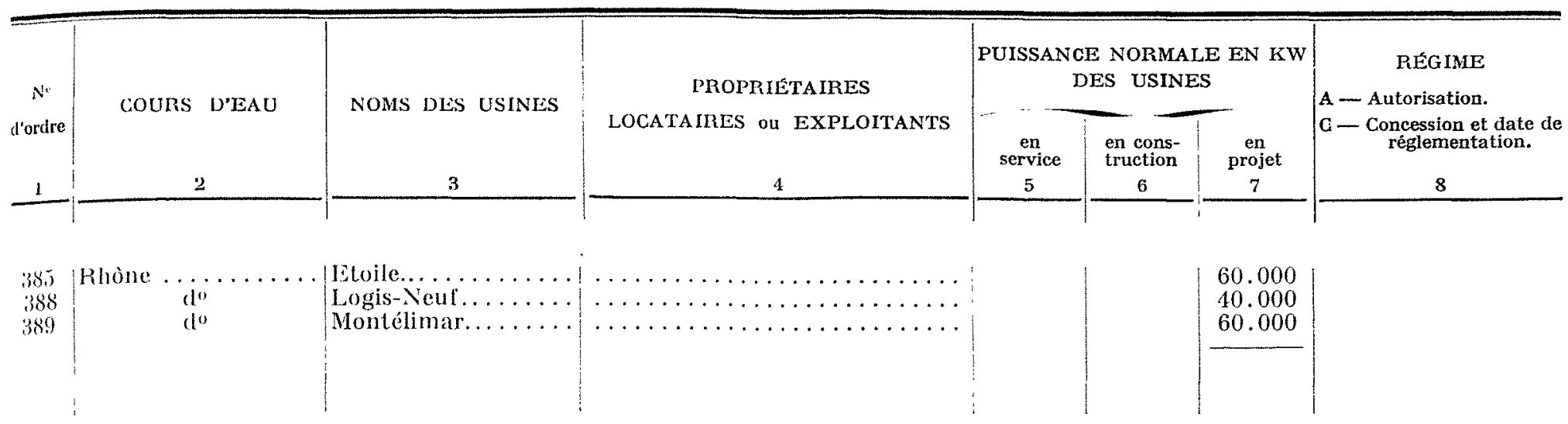

\section{BASSIN DE I'ARDËCFEE}

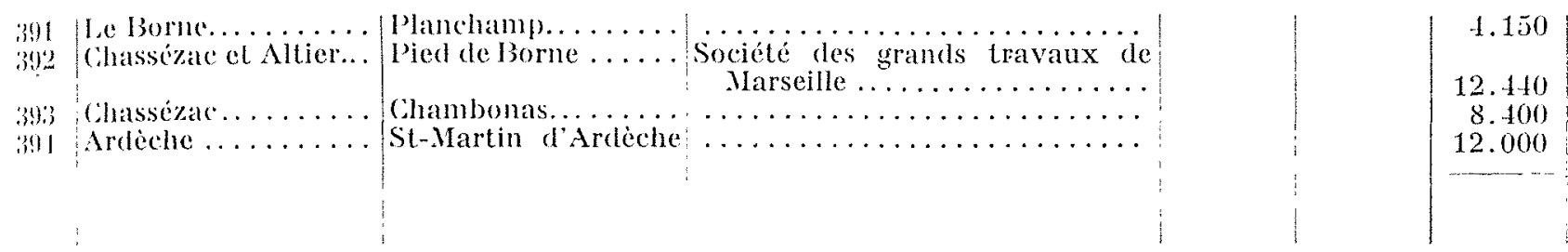

\section{BASSIN DE IA DURAINCE}

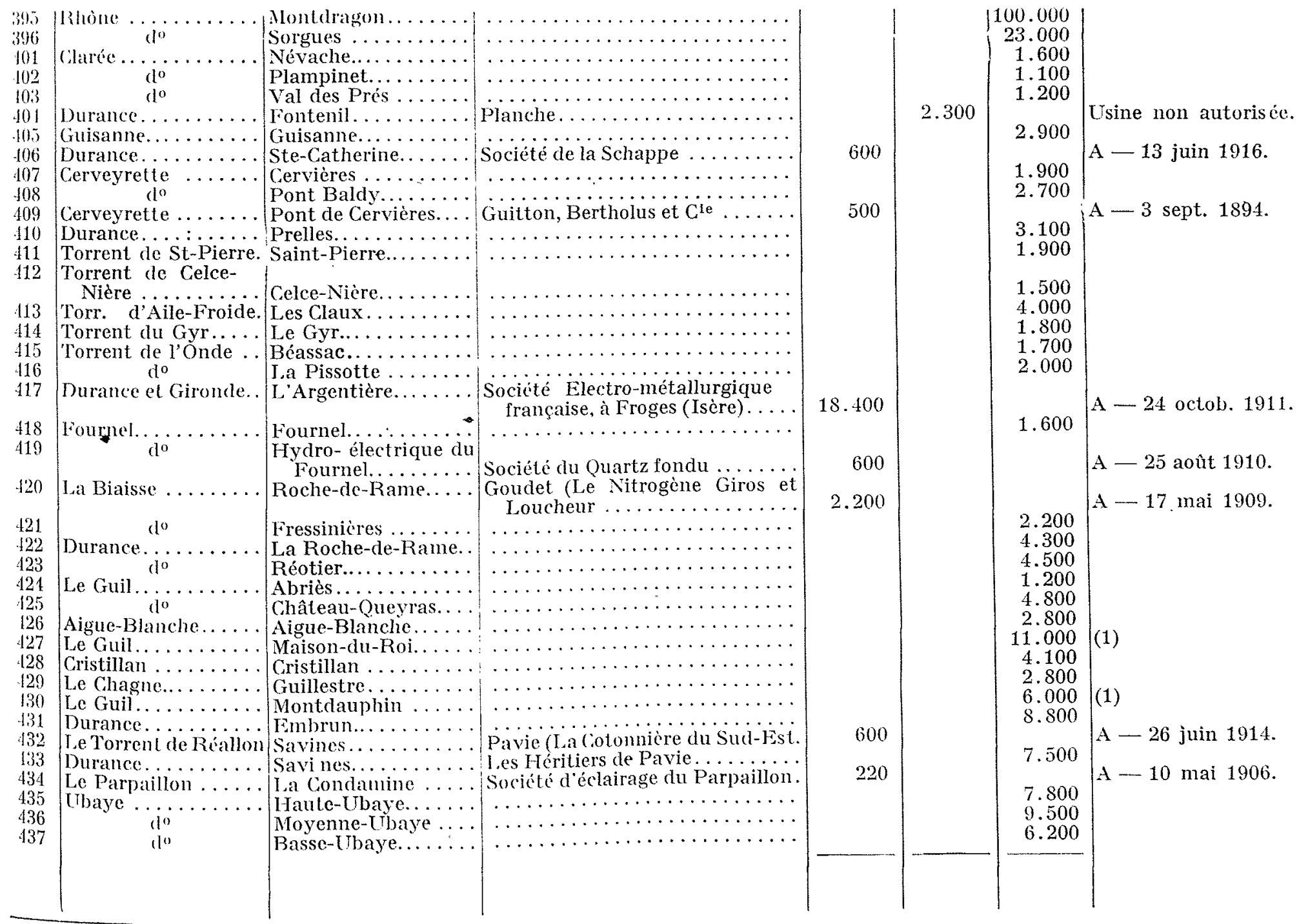

(1) Non compris la puissance due aux débits supplémentaires provenant d'accumulation. 


\begin{tabular}{|c|c|c|c|c|c|c|c|}
\hline \multirow{3}{*}{$\begin{array}{c}N^{*} \\
\text { d'ordre } \\
1\end{array}$} & \multirow{2}{*}{ COUIS D'EAU } & \multirow{2}{*}{ NOMS DES USINES } & PROPRIETAIRES & \multicolumn{3}{|c|}{$\begin{array}{l}\text { PUISSANCE NOHMALE EN ISW } \\
\text { DLS USINES }\end{array}$} & \multirow{3}{*}{$\begin{array}{c}\text { 1uEgme } \\
\mathrm{A}-\text { Autorisation. } \\
\mathrm{c}-\begin{array}{c}\text { Concession et date do } \\
\text { réglementation. }\end{array} \\
\mathbf{8} \\
\end{array}$} \\
\hline & & & LOCATAIRES OU EXPLOIMANTS & $\begin{array}{c}\text { en } \\
\text { service }\end{array}$ & $\begin{array}{l}\text { en cons- } \\
\text { truction }\end{array}$ & $\begin{array}{c}\text { en } \\
\text { projet }\end{array}$ & \\
\hline & 2 & 3 & 4 & 5 & 6 & 7 & \\
\hline
\end{tabular}

\section{BASSIN DE LA DURANCE (suite)}

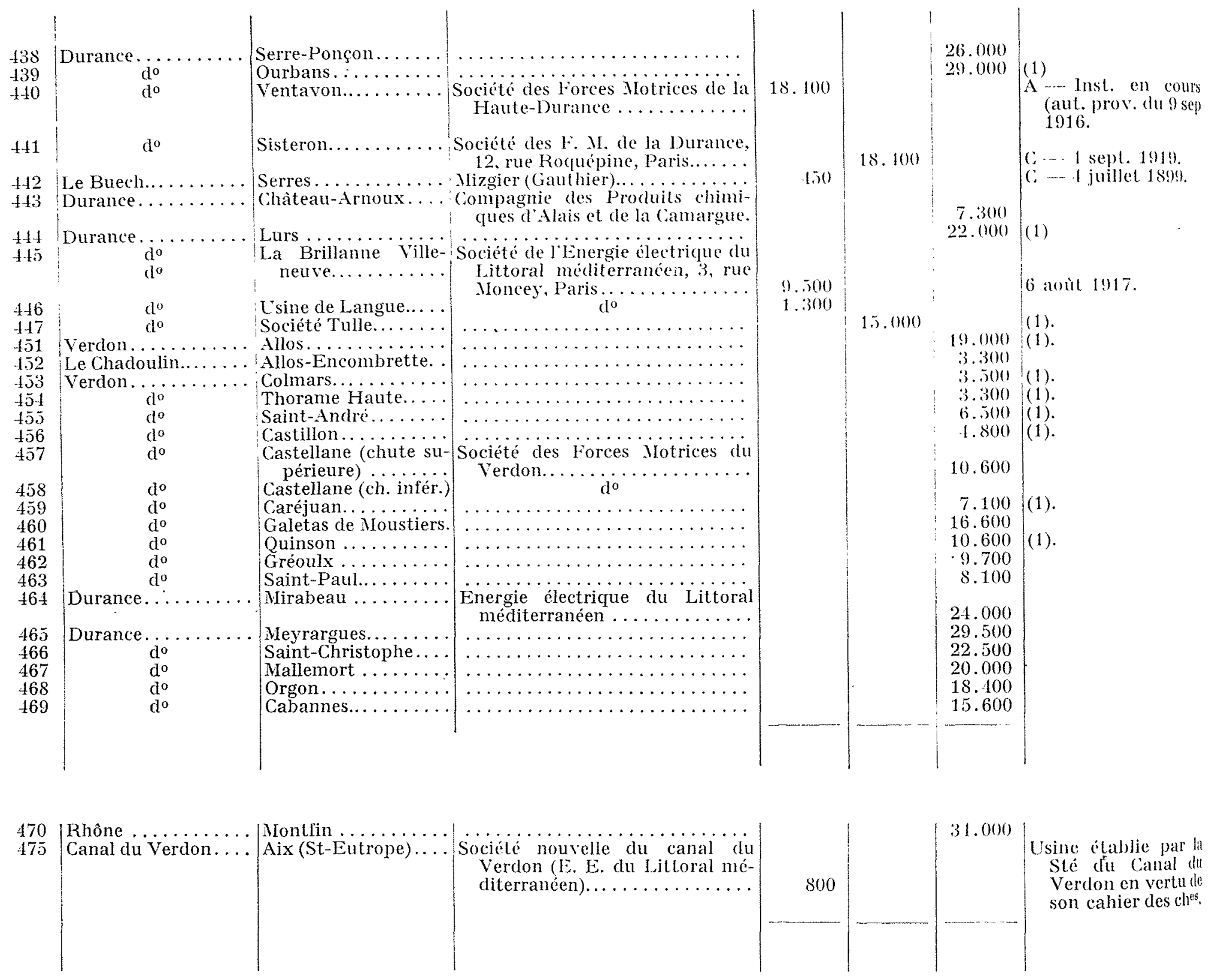

BASSIN DE IAFGEINS

BASSIN DE IA SIAGIVE 


\section{7}
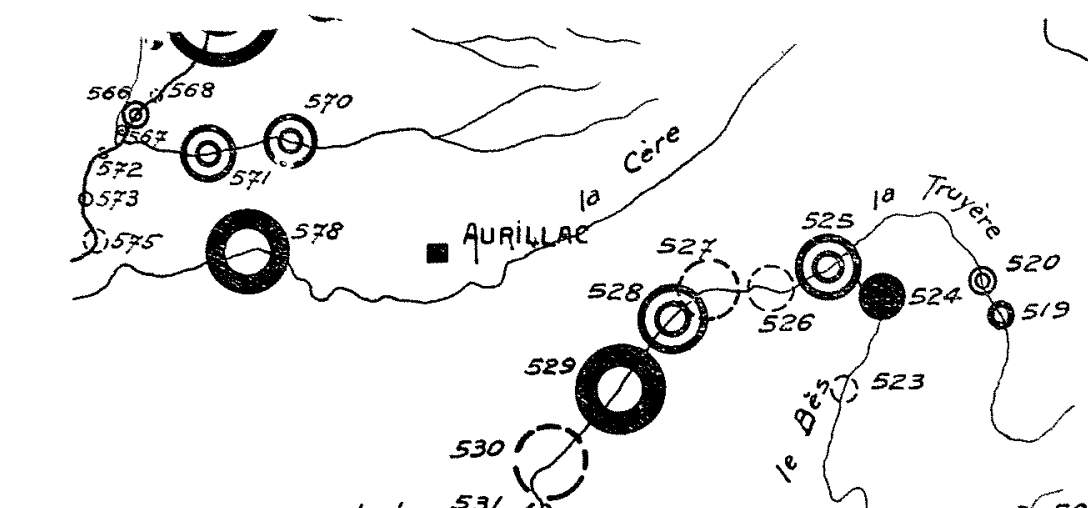

10

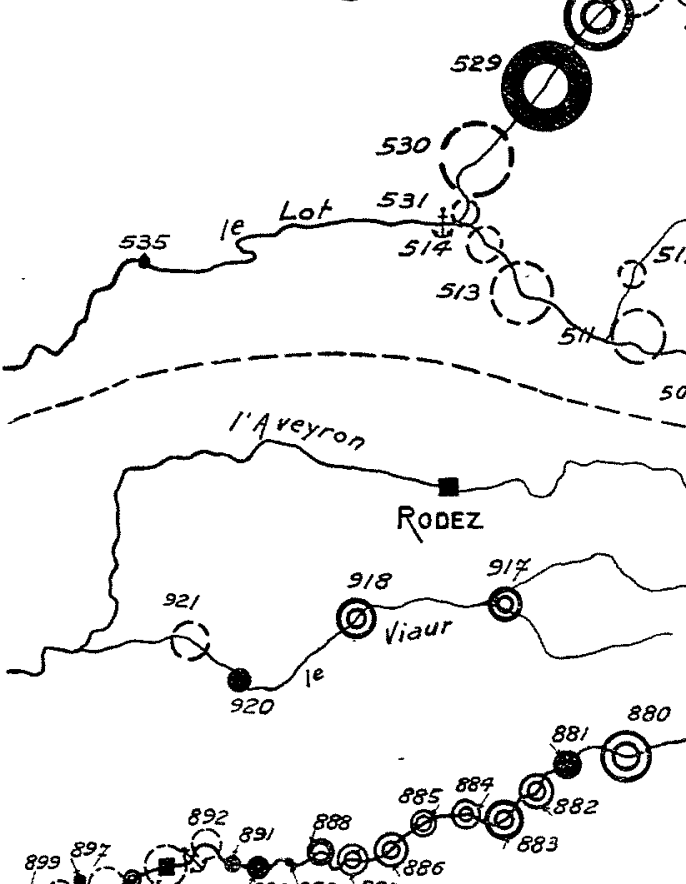

699 897

898 39895 ALBI 


\section{8}

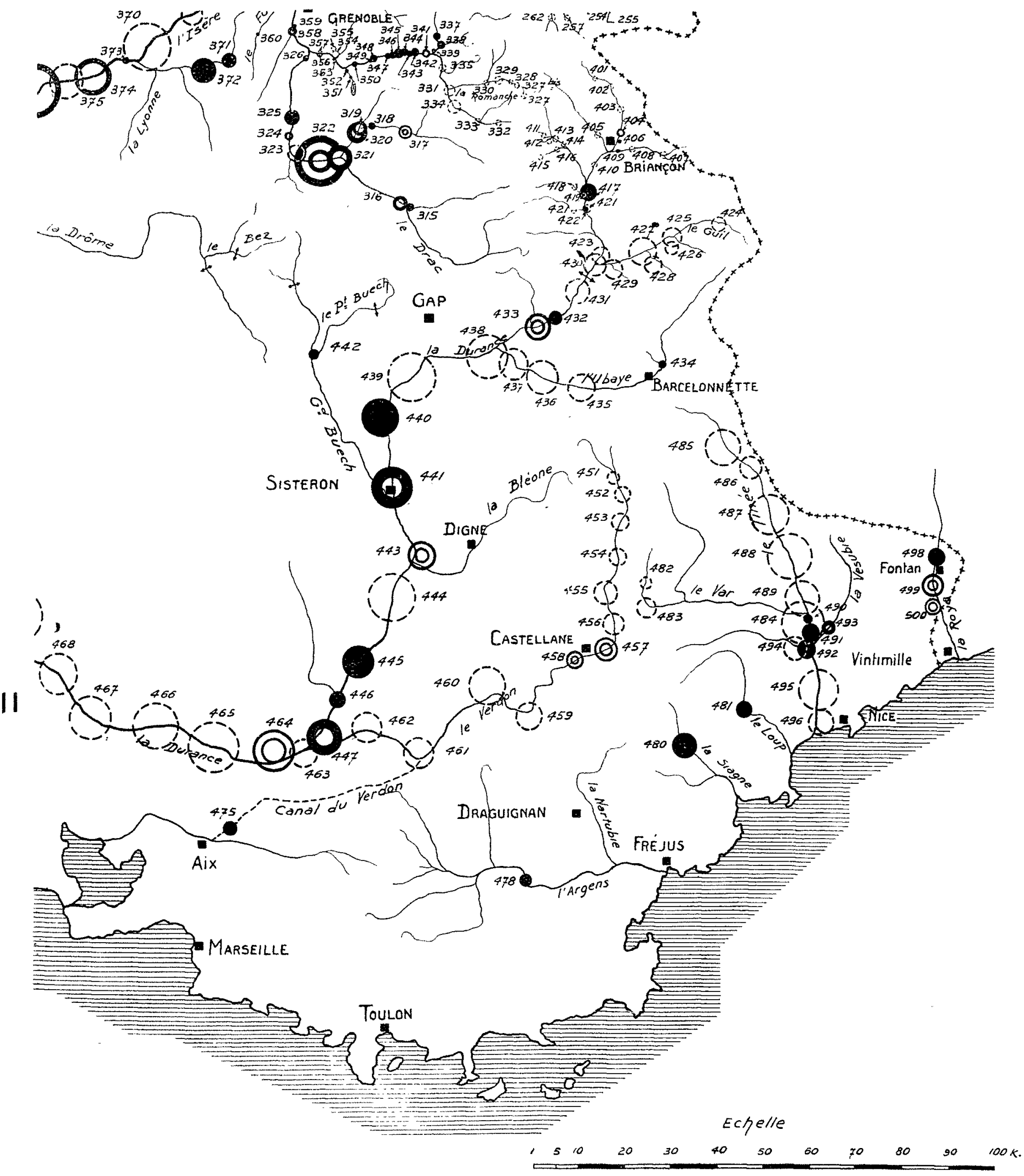

\section{LEGENOE}

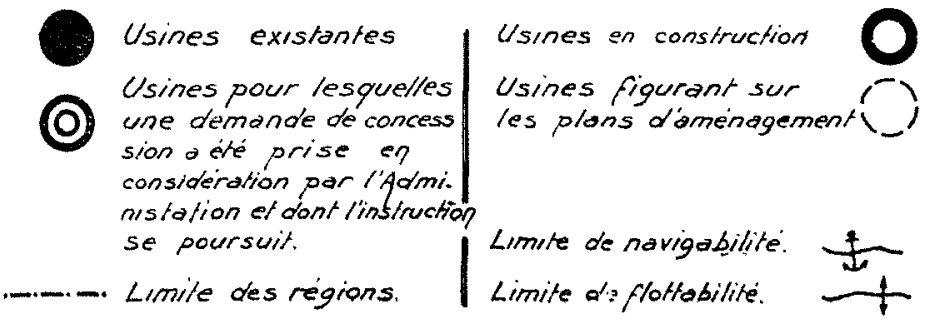




\begin{tabular}{|c|c|c|c|c|c|c|c|}
\hline \multirow{2}{*}{$\stackrel{\mathrm{N}^{\circ}}{\text { d'ordlre }^{2}}$} & \multirow{2}{*}{$\begin{array}{l}\text { COURS D'EAU } \\
2\end{array}$} & \multirow{2}{*}{ NOMS DES LisINES } & \multirow{2}{*}{$\begin{array}{c}\text { PROPHETAMRS } \\
\text { LOCATARES OU EXPLOTTANTS } \\
4\end{array}$} & \multicolumn{3}{|c|}{$\begin{array}{c}\text { PUISSANCE NORNALE EN KW } \\
\text { DLS LSINES }\end{array}$} & \multirow{2}{*}{$\begin{array}{c}\text { RFFrime } \\
\text { A-Autorisation. } \\
\text { C }- \text { Concession et date de } \\
\text { réggementation. } \\
8\end{array}$} \\
\hline & & & & 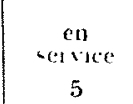 & $\begin{array}{l}\text { encon- } \\
\text { Iruction } \\
\text { on }\end{array}$ & $\begin{array}{c}\mathrm{en} \\
\text { projet } \\
7 \\
7\end{array}$ & \\
\hline
\end{tabular}

\section{BASSIN DU IOUP}

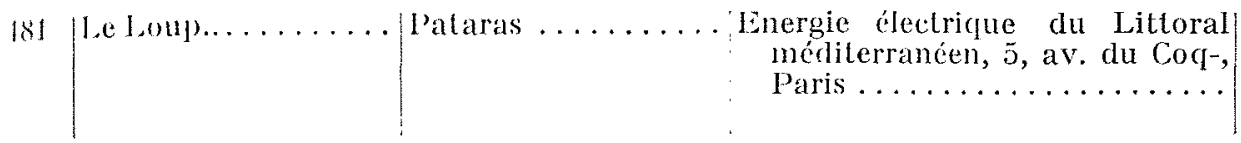

nergie électrique du Littoral

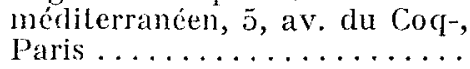

\section{BASSIN DU VAR}
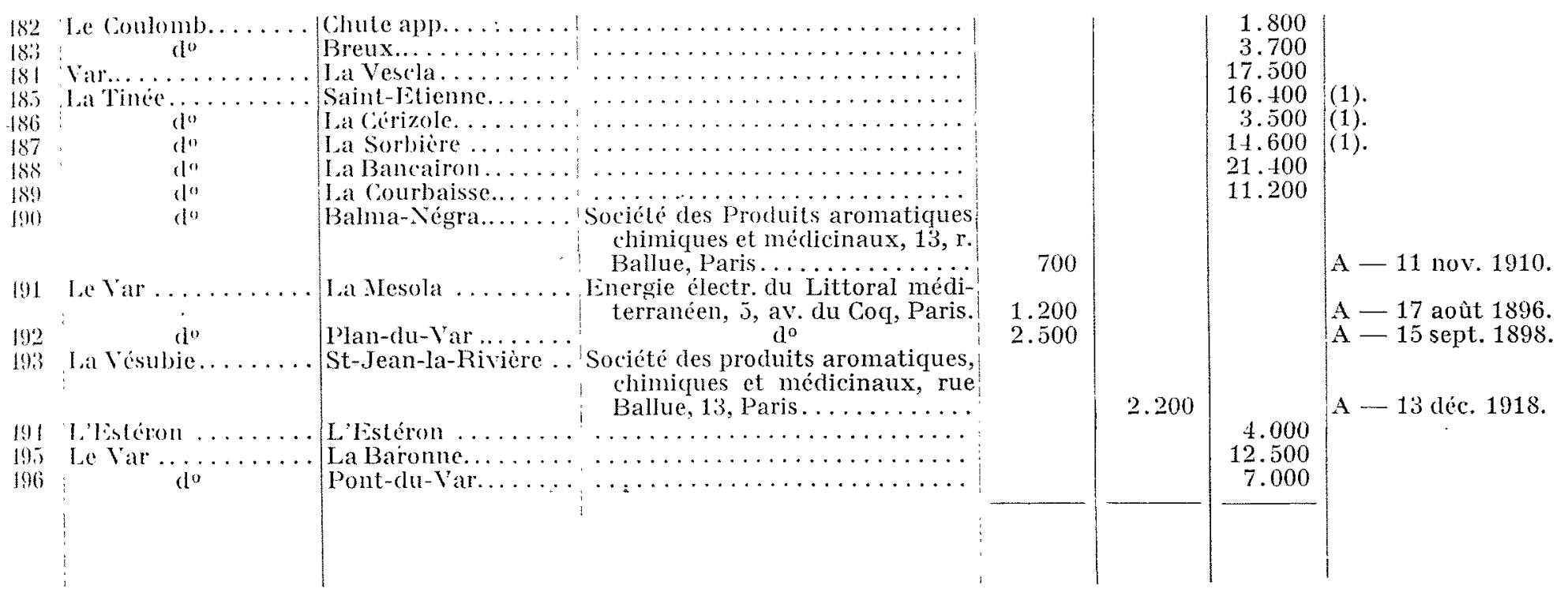

BASSIN DE I.A ROYA

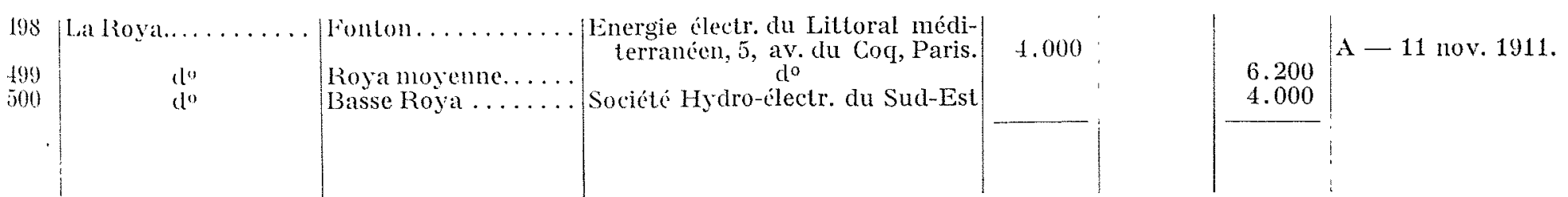

(1) Non compris la puissance due aux débils supplémentaires perdue aux 
RÉGION DU CENTRE

\begin{tabular}{|c|c|c|c|c|c|c|c|}
\hline$N^{\circ}$ & COURS D'EAU & NOMS DES USINES & 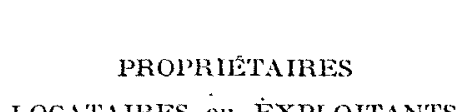 & PUISSAN & $\begin{array}{l}\text { NORMA } \\
\text { AS USIN }\end{array}$ & EN $k W$ & $\begin{array}{r}\text { REgime } \\
\text { A-Autorisation. }\end{array}$ \\
\hline 1 & 2 & 3 & 4 & $\begin{array}{c}\text { en } \\
\text { service } \\
5 \\
\end{array}$ & $\begin{array}{c}\text { cun cons- } \\
\text { truetion } \\
\quad 6 \\
\end{array}$ & $\begin{array}{c}\text { en } \\
\text { projel } \\
7 \\
\end{array}$ & $\begin{array}{l}\text { réciementatiou. } \\
8 \\
\end{array}$ \\
\hline
\end{tabular}

BASSIN DU IOT

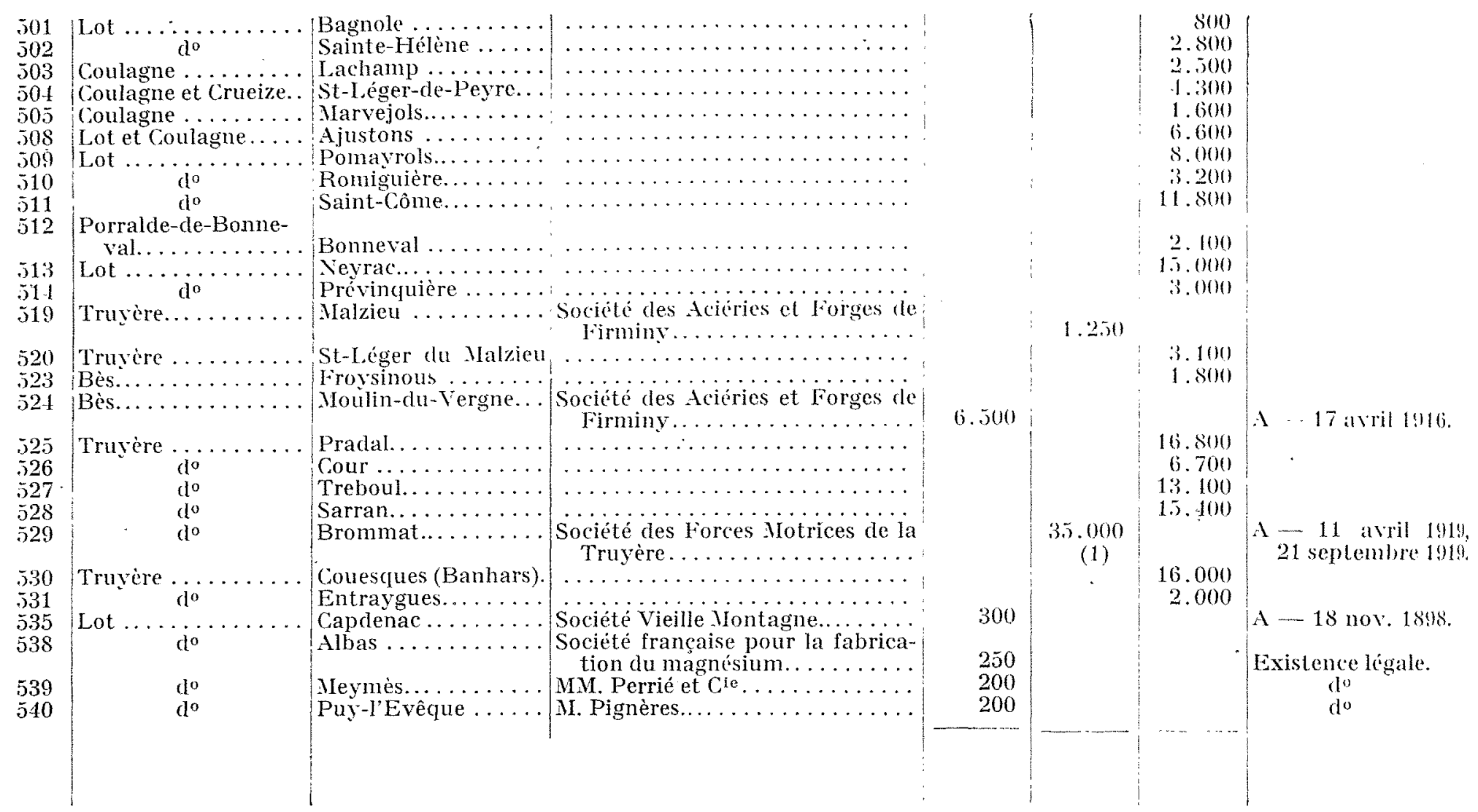

\section{BASSIN DE IA DORDOGNE}
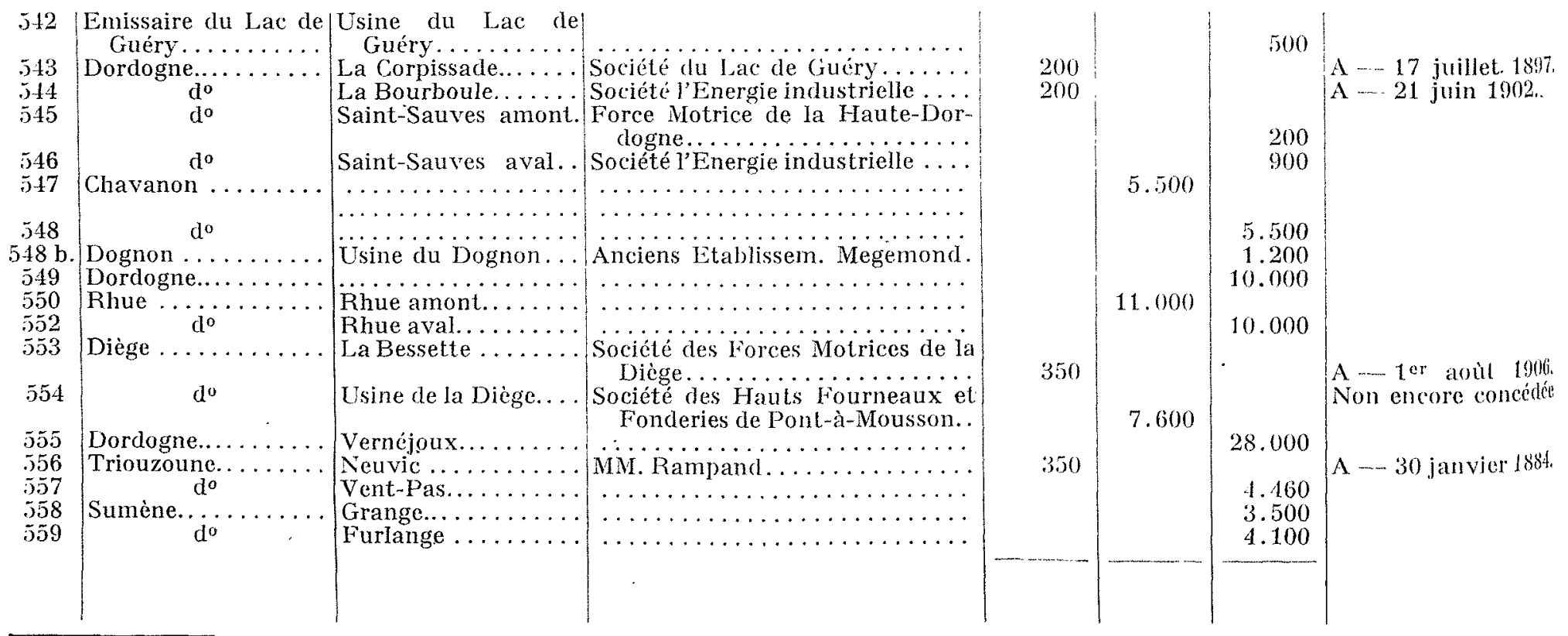

(1) Avec aménagement pour 20 mètres carrés on envisage un aménagement double et peut-être quadruple. 


\begin{tabular}{|c|c|c|c|c|c|c|c|}
\hline $\mathrm{N}^{\circ}$ & COURS D'EAU & NOMS DES USINES & PROPRIÉTAIRES & PUISSAN & $\begin{array}{l}\text { NORMA } \\
\text { AS USINI }\end{array}$ & EN KW & $\begin{array}{r}\text { RÉGIME } \\
\text { A- Autorisation. }\end{array}$ \\
\hline d'ordre & & & LOCATAIRES ou EXPLOITANTS & $\begin{array}{c}\text { en } \\
\text { service }\end{array}$ & $\begin{array}{l}\text { en cons- } \\
\text { truction }\end{array}$ & $\begin{array}{c}\text { en } \\
\text { projet }\end{array}$ & $\begin{array}{c}\mathrm{C}-\text { Concession et date de } \\
\text { réglementation. }\end{array}$ \\
\hline 1 & 2 & 3 & 4 & 5 & 6 & 7 & 8 \\
\hline
\end{tabular}

BASSIN DE LA DORDOGNE (suite)

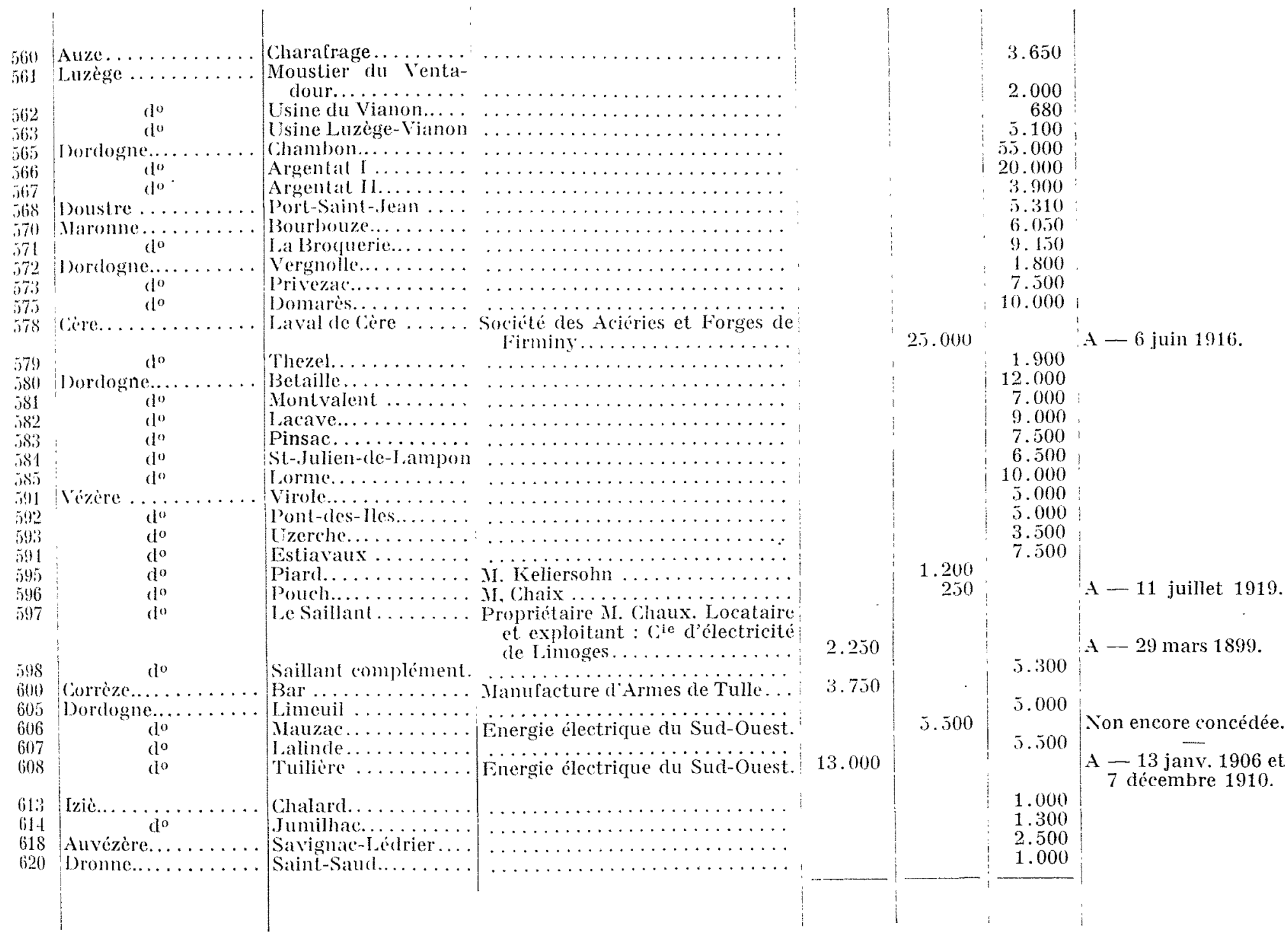

\section{BASSIN DE IA IOIRE SUPERIEURE}
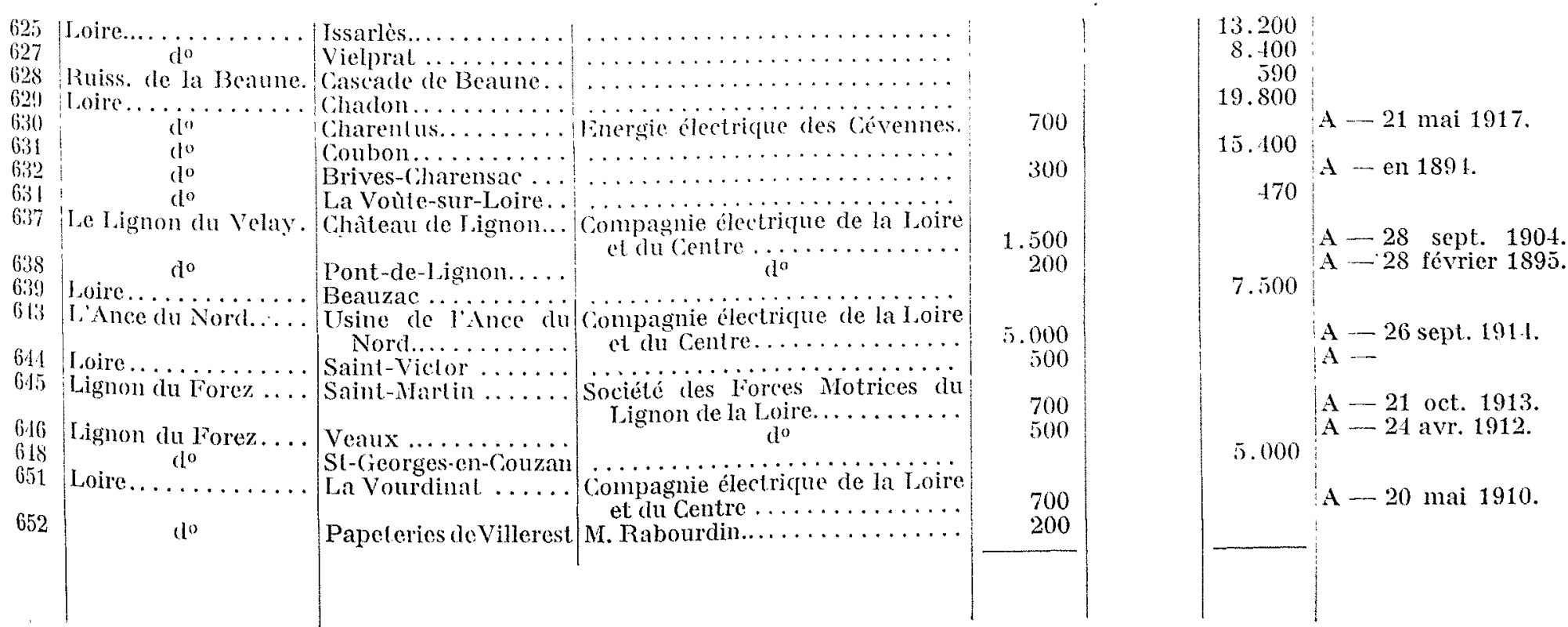


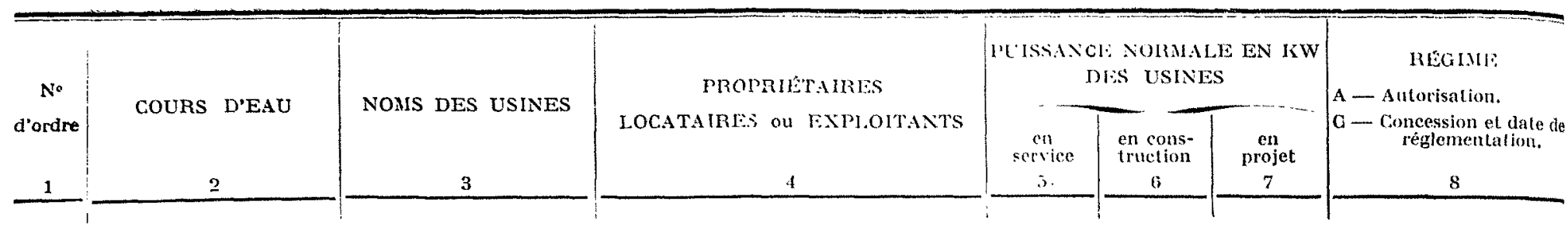

BASSIN DE I'AIIIER

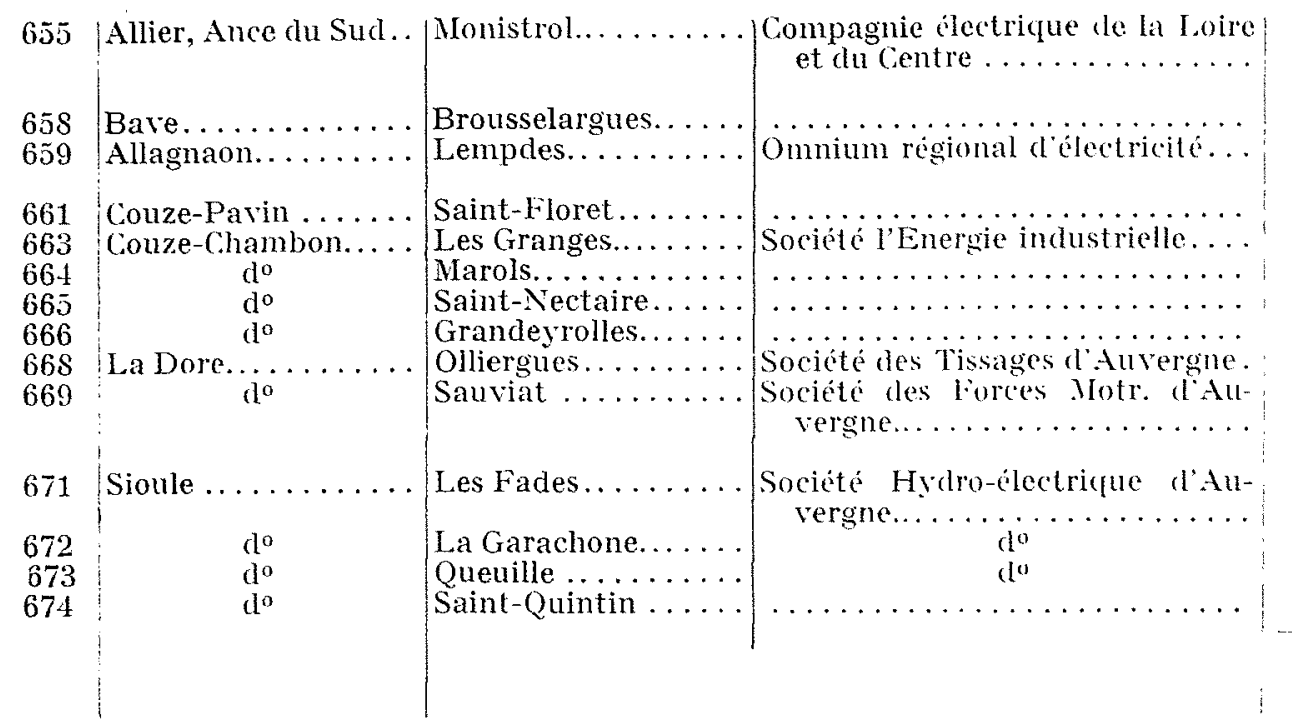

\section{BASSIN DU CFEER}

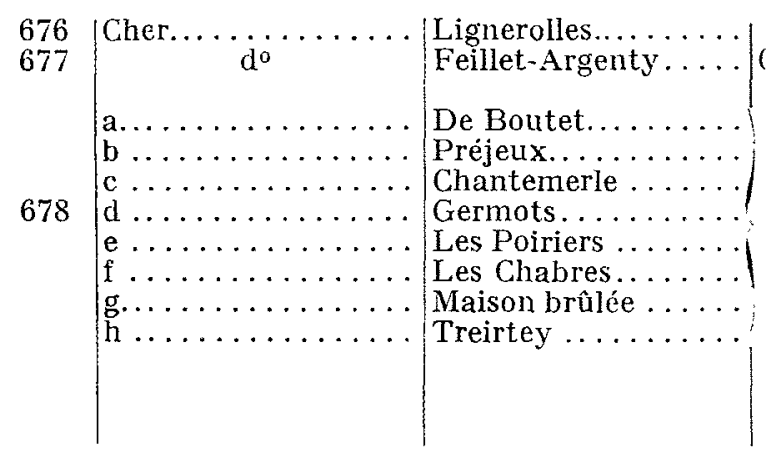

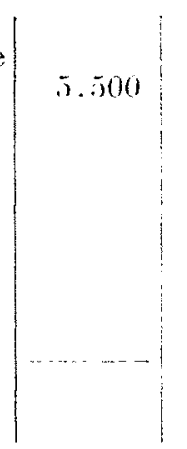

\section{BASSIIN DE IA VIEINIF}

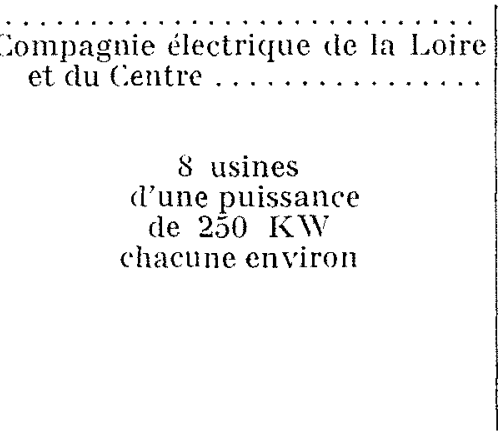

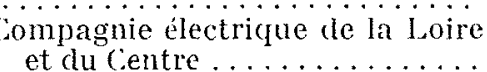

\section{8 usines}

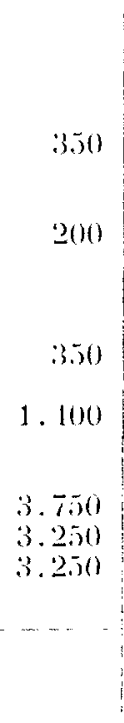

\$. 50()

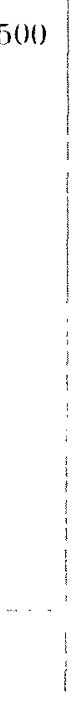

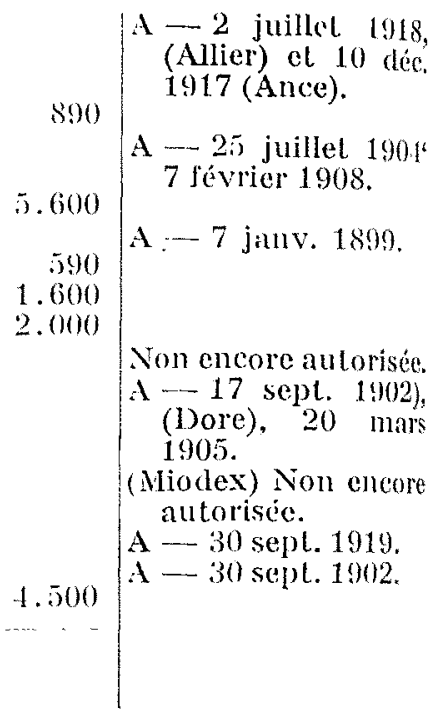

$\begin{aligned} & 1.800 \\ & 2.000\end{aligned} \mid$

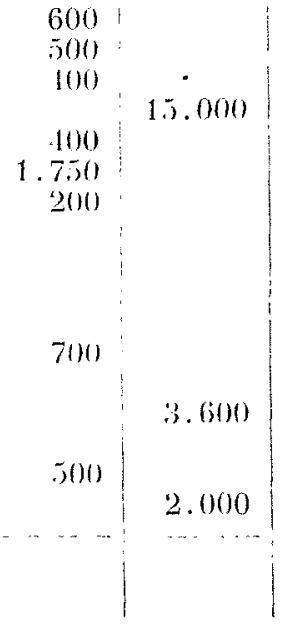

Chantemerle..........

Les Poiriers .........

Confolent............ Salandrouze .

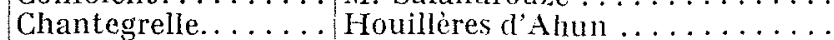

Bonnavaud ....... Société électrique de Guéret....

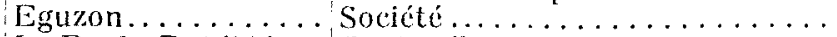

La Roche Bat l'Aigue Centre électriçue... . . . . . . . . . .

Vienne.....

Eymoutiers ....... Tramways de la Faule-Vienne..

Farebout... . . . . Papeteries du l.imousin. . . . . .

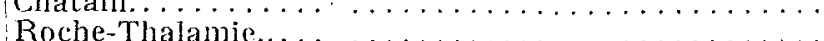

Saint-Marc....................

694

695

696

699
Le Palais ........ Société Hydro-éleclricque el Mé ciete des Forces Motrices de la Chardes.

Bonnetel-Matours....

Maisons-Rouges .... Papeteries cle la Haye-Descartes.

Tolal pour le bassin ale la vienne.
C - 12 janvier 1921

A -30 oclob. 1901 .

A -6 novemb. 1901 .

Non encore concéde

A -8 juin 1907 .

A - 25 jamvier $191 \%$

Existence lros ane

2.300

3.500

3.260

$A-22$ sept. 1006.

Non encore concédie

A - 31 janvier 1918 C - 10 avril $191 \%$. 
de faire déboucher le jet dans une chambre d'eau où l'énergie se dégradera en vagues. Un modèle au $\frac{1}{25^{\mathrm{e}}}$ représentera :

1) Une chute de $1 \mathrm{~m}, 20$;

2) Un débit de $\frac{1}{5 \times 625} \times 10 \mathrm{~m}^{3}$ : sec. $=31$ : sec. environ.

Sa réalisation est donc extrèmement simple.

Pour en terminer avec cet ordre d'applications, j'indiquerai que c'est d'après la règle précédente qu'ont été établis les modèles utilisés pour les renforceurs de chutes décrits dans la Houille Blanche, $\mathrm{n}^{\circ}$ de mars-avril 1923; et qu'il n'y aurait aucune difficulté à retrouver par une voie analogue la notion de nombre de tours spécifiques. Mais il m'a paru qu'il y avait plutôt lieu d'insister sur les applications moins classiques.

\section{VI}

Reste enfin à traiter un point de la plus haute importance au point de vue des applications :

Comment déduire des mouvements d'un modèle, les mouvements de l'appareil final ? En d'autres termes : on donne deux systèmes déformables géométriquement semblables à l'origine des temps et on demande quelles relations doivent exister entre les forces appliquées aux diverses parties des deux systèmes, entre les masses des parties correspondantes, etc., pour que la forme du système $\mathrm{S}$ au temps $t$, soit la même que celle du système $S$ ' au temps $t$,' lorsque l'on a :

$$
\frac{t}{t^{\prime}}=\mathrm{Ct}
$$

J'indique sur un cas particulier, schématisant ce qui se produit dans certains déchargeurs, la méthode employée ; elle est d'ailleurs générale.

Soit un cylindre fermé par un piston de masse $m:$ un ressort $R$ repousse le piston vers la droite; une pression $p$ refoule le piston

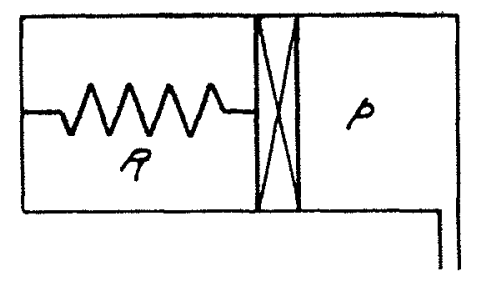

vers la gauche. Soit $x$ une variable représentant le déplacement du piston vers la gauche; nous supposerons que $p$ est fonction de $x$.

L'équation générale du monvement du piston est, en appelant $k$ la constante du ressort :

$$
m \frac{d^{2} x}{d l^{2}}+k x-p S-0
$$

Cela montre que $x$ dépend du temps $t$, de l'élasticité $k$ du ressort, de la pression $p$, de la masse $m$, d'une grandeur $l$, représentant à l'échelle d'exécution, une dimension caractéristique (dans l'équation actuelle, $l$ intervient par le facteur $\mathrm{S}=C \mathrm{C}^{2}$ ).

Ecrivant donc :

$$
x=/(m, l, k, p, l)
$$

et développant en série, on obtient pour le terme général :

$$
\text { A } m^{\alpha} \ell^{\xi} k \gamma p^{\delta} l^{c}
$$

Donc :

$$
\begin{aligned}
& {[x]=M^{\alpha} T^{\hat{\gamma}}\left(M T^{2}\right)^{\gamma}\left(\mathrm{L}^{-1} \mathrm{MT}^{-2}\right)^{\hat{\delta}} \mathrm{L}^{\mathrm{z}}} \\
& 1=\varepsilon-\delta \quad . \varepsilon=1+i \\
& 0=x+\because+2
\end{aligned}
$$

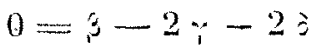

$$
\begin{aligned}
& x=\cdots-\because-\frac{\partial}{\partial} \\
& \beta=2 i+2 j
\end{aligned}
$$

Et le terme général devient :

$$
\text { A }\left\lceil\frac{l^{2} k}{m}\right\rceil i\left[\frac{l^{2} p l}{m}\right\rceil^{i}
$$

En d'autres termes, on a :

$$
\left.i=1 \mid \frac{m}{k l^{2}}, \frac{m}{p l l^{2}}\right\rfloor
$$

Le deplacement relatif $\frac{x}{l}$ sera donc le mème quand on aur entre le système et son modèle les relations :

$$
\begin{aligned}
\frac{m}{k t^{2}} & =\frac{\mathrm{M}}{\mathrm{KT}^{2}} \\
\frac{m}{p l t^{2}} & =\frac{\mathrm{M}}{\mathrm{PLT}^{2}}
\end{aligned}
$$

Ou encore:

$$
\begin{aligned}
\frac{m}{k t^{2}} & =\frac{\mathrm{M}}{\mathrm{KT}^{2}} \\
\frac{p l}{k} & =\frac{\mathrm{PL}}{\mathrm{K}}
\end{aligned}
$$

Supposons que l'on ait:

$$
\begin{aligned}
& \frac{l}{\mathrm{~L}}=\frac{1}{\alpha}\left(\text { modêle à l'échelle } \frac{1}{\alpha}\right) \\
& \left.\frac{p}{\mathrm{P}}=\frac{1}{\beta} \text { (pression réduite dans le rapport de } 1 \text { à } \hat{\beta}\right)
\end{aligned}
$$

et que l'appareil et son modèle soient de mème matière $\left(\frac{m}{M}=\frac{1}{x^{3}}\right.$ on aura:

et

$$
\frac{k}{\mathrm{~K}}-\frac{1}{2}
$$

$$
\begin{gathered}
\frac{t^{2}}{\mathrm{~T}^{2}}=\frac{m}{\mathrm{M}} \times \frac{\mathrm{K}}{k}=\frac{\dot{i}}{x^{2}} \\
\frac{l}{\mathrm{~T}}=\frac{1}{*} V
\end{gathered}
$$

Prenant pour fixer les idées $\%=\%=10$, on voit que la simi litude dynamique exige :

1) Que l'élasticité des ressorts du modèle soit 100 fois plu faible que l'élasticité des ressorts récls.

2) Que les temps du modèle soient comptés à une échelle 1 i1 fois plus petite que les temps de l'appareil réel : les mouvement du modèle sont donc plus rapides.

\section{VII}

J'ai essayé, dans l'article précédent, d'exposer les principes th la similitude dynamique, principes indispensables à connaitrt pour qui veut réduire le nombre des essais, c'est-à-dire en définilive le coût des résultats. La méthode suivie consiste en sommi à effectuer une analyse préliminaire des différents facleurs d'artion, à déterminer ceux que l'on peut écarter à priori. On pourra parfois hésiter dans cette voie ; il semblera difficile de dénier de prime abord un caractère d'importance à tel ou tel élément, ou, au contraire, d'introduire un facteur dissimulé, mais la difllculté est au fond idenlique à celle qui se pose lors de la mise ell equations d'un problème technique quelconque. Le sens des rétlités doit alors intervenir et l'expérience surtout rloit juger ent dernier ressort. 